Monika KULESZA

Université de Varsovie

\title{
MADAME DE SÉVIGNÉ ET LES THÉORIES DU ROMAN
}

$\mathrm{Au} \mathrm{XVII}^{\mathrm{e}}$ siècle le roman jouit d'un statut à peine littéraire. Il est dénigré par les doctes, car Aristote n'en parle pas dans sa Poétique, mais il remporte un succès considérable auprès des mondains et surtout auprès des mondaines qui non seulement lisent les romans, mais en sont parfois les auteurs. Lire les romans, écrits par et pour les membres du même cercle, fait partie du mode de vie de la société mondaine.

Certes, Mme de Sévigné n'a jamais écrit de roman, mais elle était une lectrice assidue de fictions romanesques. De même elle ne s'est jamais spécialisée dans la critique littéraire et pourtant l'on trouve dans ses lettres les remarques qui témoignent de l'intérêt qu'elle portait à la lecture et surtout du goût raffiné pour la littérature. Tout comme pour d'autres domaines, le théâtre par exemple ${ }^{1}$, les jugements de la marquise ne sont pas exprimés arbitrairement, mais adroitement glissés dans le contenu de la lettre. Il faut donc relever ces fragments qui prouvent que l'épistolière était une lectrice cultivée, attentive et toujours au courant des nouveautés relatives au genre romanesque.

La variété de ses lectures, les guides savants qui l'aidaient à se former ainsi que le fait de connaître les auteurs et les œuvres où ils exposaient leurs réflexions sur l'idéal romanesque constituent une précieuse source d'information sur les théories du roman connues de Mme de Sévigné.

Les lectures de notre épistolière étaient très diverses: romans, pièces de théâtre, fables, poèmes, mémoires, portraits, oraisons funèbres, sermons, ouvrages historiques, traités philosophiques et religieux, la Bible... « Nous lisons beaucoup, écrit-elle le 29 décembre 1675, et du sérieux, et des folies, et de la fable et de l'histoire » (II, 205) ${ }^{2}$. L'épistolière lit les œuvres de Pétrarque, de Guarini, de l'Arioste et du Tasse, apprécie tout particulièrement La Fontaine et Boileau et, quand elle est plus âgée, Bossuet, Pascal ou la Morale de Pierre Nicole. Elle se passionne pour les romans et elle aime relire ceux de sa jeunesse: Cassandre, Cléopâtre, Pharamond écrits par de La Calprenède ainsi qu'Artamène ou Le Grand Cyrus et Clélie de Mlle de Scudéry. La marquise, comme beaucoup de ses contemporains, lisait les romans en quelque sorte en cachette, sans l'avouer, puisqu'ils étaient considérés comme une littérature médiocre, à lire « aux heures perdues. C'est ordinairement sur cette lecture que je m'endors, le caractère m'en plaît beaucoup plus que le style. Pour les sentiments, j'avoue qu'ils me plaisent aussi et qu'ils sont d'une perfection qui remplit mon idée sur les belles âmes. Vous savez aussi que je ne hais pas les grands coups d'épée [...] pourvu qu'on m'en garde le secret » (15. VII. 1671, I, 296).

\footnotetext{
${ }^{1}$ Cf. KulesZa (1998).

${ }^{2}$ Mme de Sevigne (1972-1978). Toutes les lettres sont citées d'après cette édition et les citations sont suivies entre parenthèses de la date (sauf quand elle est indiquée dans le texte), du numéro du volume et de la page.
} 
Lectrice passionnée de romans, à l'affût du romanesque dans la vie tout autant que dans les œuvres, Mme de Sévigné est contemporaine de l'émergence en France de ce nouveau genre qu'est le roman moderne. Elle vit dans une époque de grande créativité artistique où l'on est aussi fort soucieux de fixer des normes et des interdits. Le roman a ses théoriciens et tout un chacun, dans le beau monde, se pique d'en être. Mme de Sévigné appartient à ce milieu, grâce à sa situation privilégiée, celle d'une femme qui dans sa jeunesse fréquentait l'Hôtel de Rambouillet « dont elle a tiré, entre autres et peut-être surtout, un commerce suivi avec le cercle qui constitua l'héritage le plus lettré de ce salon, celui de Mme de La Fayette et de La Rochefoucauld » (VIALA, 1996 : 62). L'abbé de Pure, dans sa Prétieuse ou le mystère des ruelles (1656-1658), relate les conversations qui se tenaient dans les cercles de précieuses où l'on discutait de littérature. L'on y débattait, entre autres, de la vraisemblance romanesque, l'on examinait la psychologie du cœur humain et l'on se penchait sur l'opposition entre le désir et le plaisir, « le plaisir de l'attente et de la curiosité, causé par les trouvailles originales de l'auteur, [aussi bien] que [sur] le plaisir du dénouement heureux » (COULET, 1968 : 72). Tandis que certaines lectrices désirent le roman galant «qui est un Roman de pur amour » (COULET, $1968: 71$ ), d'autres croient « qu'il ne peut estre que fade et insipide, s'il n'y a point de mélange de bravoure et de valeur» (COUlET, $1968:$ 73).

Mme de Sévigné a participé, ou au moins assisté, à ce genre de conversations au cours desquelles les femmes exprimaient leurs goûts en matière de roman. Grâce à ces fréquentations ainsi qu'à ses liens de parenté (surtout avec BussyRabutin dont les connaissances en matière de littérature et de langue ne faisaient aucun doute aux yeux de ses contemporains), elle bénéficiait de relations avec des érudits capables de la guider dans ses lectures et qui l'accompagnaient dans son apprentissage littéraire.

C'est par Gilles Ménage, un spirituel abbé dont elle partageait l'amitié avec Mme de La Fayette que «Mme de Sévigné fut en contact avec la mouvance de Foucquet, donc avec Pellisson, Scudéry, Sarasin, Guez de Balzac, Scarron, La Fontaine; au-delà, le réseau s'étendait aux mondains "galants », proches de l'influence jésuite» (VIALA, 1996 : 62). Ménage était un auteur ${ }^{3}$ à la verve polémique et son goût pour les belles lettres le rendait expert aux yeux des mondains. L'abbé faisait connaître aux deux dames certains livres, tels que les tomes III et IV de Clélie ou « la onzième lettre des jansénistes » ${ }^{4}$ (12. IX. 1656, I, 41). Ils entretenaient tous trois un commerce littéraire ${ }^{5}$ quand la marquise était aux Rochers. Le meilleur exemple de leurs échanges littéraires est «l'affaire des madrigaux $»^{6}$. Pour la rappeler brièvement: Ménage a composé en italien un madrigal imitant celui écrit en français par Raincy ; il a attribué le sien au Tasse et donné les deux à juger à Chapelain, sans l'informer du subterfuge. Il a découvert par la suite sur le même thème un autre madrigal, de Guarini celui-là. Non content

\footnotetext{
${ }^{3}$ Notamment Origines de la langue française (1650), et de nombreux poèmes comme celui intitulé «Le Pêcheur ou Alexis. Idylle à Madame la marquise de Sévigné » dans Miscellanea (1652).

${ }^{4}$ La $11^{\mathrm{e}}$ Provinciale de Pascal.

${ }^{5}$ Cf. aussi les lettres de Mme de La Fayette, le 26 octobre et le 2 novembre 1655 dans Mme de LA FAYETTE (1942: 56-59).

${ }^{6}$ Mme de Sevigne (1972-1978), Notes, ${ }^{0} 5$, I, 872.
} 
d'avoir mystifié Chapelain, il a voulu connaître l'avis de ses deux correspondantes sur les trois poèmes. La marquise a hésité, mais, finalement, elle a choisi le vrai Guarini et non pas le faux Tasse sans qu'elle «puisse quasi dire pourquoi » (à Ménage, 12. IX. 1656, I, 39). «Tel est le style des relations de Ménage et de ses correspondantes : il les instruit par la pratique, en les invitant à choisir et à justifier leur avis » (DUCHENE, $1982: 113)$.

Le second mentor qui contribua à exercer l'esprit et à former le goût de la marquise était justement Jean Chapelain. Ami de sa famille, il introduisit Mme de Sévigné à l'Hôtel de Rambouillet où il régnait en maître, même après l'échec de sa Pucelle. C'est aussi grâce à lui que la marquise a fréquenté un autre cercle, celui du Cardinal de Retz et de Boileau, proche de la mouvance janséniste. Son rôle auprès de la marquise était de même nature que celui joué par Ménage : former son goût par la pratique et l'échange.

La lettre de Chapelain, datée du 19 novembre 1663, illustre bien les rapports de l'érudit et de Mme de Sévigné. Elle était à ce moment-là à Livry, lui à Paris. Leur discussion épistolaire porte sur La Jérusalem délivrée du Tasse. « Je vous l'ai dit, Madame, il y a plus d'un an et vous le dis encore à cette heure que, dans ces quatre derniers vers [de La Jérusalem] du Miroir d'Armide, Le Tasse, à force de vouloir être aigu, s'est émoussé et est tombé dans un franc galimatias qui ne lui fait pas tout l'honneur du monde, surtout pour les deux derniers vers » (I, 54-55). Et Chapelain d'expliquer le sens des vers italiens. Dans la dernière phrase il rappelle à sa correspondante que critiquer un passage ne signifie pas dénigrer l'œuvre entière.

Par ailleurs, Chapelain est auteur de la préface de l'Adone de Marino (1623), dans laquelle il définissait la poésie épique moderne. C'est pour cette raison que Mme de Grignan a demandé à sa mère de le consulter sur un passage de l'Adone. La marquise lui répond, le 24 février 1672 : «M. Chapelain a reçu votre souvenir avec enthousiasme. Il dit que l'Adone est délicieux en certains endroits, mais d'une longueur assommante. Le chant de la comédie est admirable. Il y a aussi un petit rossignol qui s'égosille pour surmonter un homme qui joue du luth. Il se vient percher sur sa tête, et enfin il meurt; on l'enterre dans le corps du luth. Cette peinture est charmante $»$ (I, 445).

L'avis de Chapelain était très écouté. Mme de Sévigné était donc contente de recevoir la réponse de l'auteur de la Pucelle lui confirmant la bonne interprétation du passage qui avait posé un problème à Mme de Grignan. Les jugements littéraires des deux dames, fondés seulement sur le goût et le plaisir, se trouvaient finalement en harmonie avec le point de vue du théoricien.

De tous les romans, c'est La Princesse de Clèves qui sans conteste apparaît le plus souvent dans la correspondance de la marquise. Elle mentionne l'existence du roman six ans avant sa parution, mais sans le nom de Mme de La Fayette (I, 459). C'est surtout entre mars et novembre 1678 qu'il est question de ce roman dans la Correspondance, l'époque où La Princesse de Clèves a suscité une très vive discussion. Les lettres de Bussy ${ }^{7}$ apportent un éclaircissement important à celles de la marquise, qui a toujours fait attention à ce qu'elle disait et elle aimait se réfugier derrière l'autorité d'autrui et surtout d'un homme qu'elle considérait comme savant et ayant bon goût. Après avoir lu ce que Bussy pensait du roman à

\footnotetext{
${ }^{7}$ Surtout celles du : 22. III. 1678, II, 604 ; 26.VI. 1678, II, 617 ; 27. VII. 1678, II, 618.
} 
la mode, elle déclare, le 27 juillet 1678 : «Votre critique de La Princesse de Clèves est admirable, mon cousin. Je m'y reconnais, et j'y aurais même ajouté deux ou trois petites bagatelles qui vous ont échappé. [...] J'ai été fort aise de savoir votre avis, et encore plus de ce qu'il se rencontre justement comme le mien ; l'amour propre est content de ces heureuses rencontres » (II, 618-619).

Il est regrettable que la marquise n'ait pas précisé ces bagatelles : cela nous aurait fait connaître son analyse critique de l'un des rares romans de son époque qui illustre encore aujourd'hui la littérature du XVII ${ }^{\mathrm{e}}$ siècle.

Mme de Sévigné mentionne aussi la critique du roman faussement attribuée au P. Bouhours ${ }^{8}$ (II, 636) ce qui prouve qu'elle était parfaitement au courant des idées critiques concernant l'œuvre de sa meilleure amie.

La plupart des romans étaient précédés d'avertissements aux lecteurs dans lesquels les auteurs expliquaient leurs intentions, les difficultés auxquelles les lecteurs allaient être confrontés, et, avant tout, ils y exposaient leur avis sur la construction de l'œuvre, sur les sujets romanesques et sur la façon d'écrire un roman. Bien entendu la pratique ne correspondait pas forcément aux règles préconisées, mais la future évolution du genre était ainsi tracée.

Nous pouvons donc supposer que c'est grâce aux préfaces que Mme de Sévigné se tenait au courant des théories du roman. Comme d'habitude, elle ne discute pas les idées des auteurs, elle mentionne seulement la lecture de l'œuvre. Mais puisqu'elle affirme, à divers endroits, lire toujours les ouvrages entiers, nous pouvons en déduire qu'elle appréciait aussi les avertissements.

Ainsi, familière de Mlle de Scudéry, la marquise connaissait les idées de Georges de Scudéry, exprimées notamment dans Ibrahim. Le romancier croit fermement aux règles, surtout à celle qu'impose la vraisemblance : un héros doit accomplir des actions hérö̈ques, mais il faut veiller à ce que «ces actions incroyables [ne] dégenerent en contes ridicules. [...] Entasser avantures sur avantures, sans ornemens, et sans exciter les passions par les artifices de la Rethorique [...] est plus d'une vieille Chronique que d'un Roman. » (COULET, 1968 : 46). Toujours selon lui, les héros doivent exprimer leurs sentiments et leurs jugements par leurs propres paroles. L'on parle des exploits sans les montrer. La bienséance doit absolument être préservée pour ne pas offenser les lectrices, le roman est une étude de l'âme humaine et « le stile narratif ne doit pas estre trop enflé, non plus que celuy des conversations ordinaires; que plus il est facile plus il est beau ; qu'il doit couler comme les fleuves et non bondir comme les torrens » (COULET, 1968 :48).

Toujours fidèle dans ses lettres au style naturel et agréable, la marquise ne pouvait qu'apprécier les principes aussi clairs qu'elle pouvait, ne serait-ce qu'inconsciemment, adopter dans ses missives : simples et très expressives à la fois.

Les idées de la sœur sont aussi familières à Mme de Sévigné car les deux dames échangent les lettres et le nom de Mlle de Scudéry apparaît fréquemment dans la correspondance. En 1654, dans l'Avis au lecteur de Cyrus, la romancière se prononce à son tour sur les principes qui doivent guider l'auteur d'une œuvre : «Vous pourrez, dis-je, voir qu'encore qu'une Fable ne soit pas une Histoire et

\footnotetext{
${ }^{8}$ Les Lettres à Mme la marquise*** sur le sujet de la Princesse de Clèves sont en fait de JeanBaptiste du Trousset de Valincour.
} 
qu'il suffise à celuy qui la compose, de s'attacher au vray-semblable, sans s'attacher toûjours au vray » (SCUDERY, $1654: 5$ ). Vingt ans après (en 1661), dans Clélie (tome X), elle développe certaines idées de son frère. Ce qui l'intéresse ce sont les rapports entre la fiction et la vérité. Elle affirme que «le veritable art du mensonge est de bien ressembler à la verité » (SCUDERY, 1682 : 39) ; le romancier doit veiller à ce que les actions des héros soient conformes au caractère et au tempérament dont il les a dotés et à ce « que les passions y soient bien depeintes ; que les avantures soient naturelles et sagement inventées; que toutes les petites choses qui font connoître le fond du cœur de tous les hommes y soient placées à propos ; que le vice y soit blâmé, et la vertu recompensée » (SCUDERY, 1682 : 46).

A part les romans de Mlle de Scudéry, Mme de Sévigné mentionne aussi les Conversations dans lesquelles Sapho reprend la plupart des passages théoriques de Cyrus et de Clélie : "Mlle de Scudéry vient de m'envoyer deux petits tomes de Conversations; il est impossible que cela ne soit bon, quand cela n'est point noyé dans son grand roman » (29. IX. 1680, III, 27).

C'est ainsi que Mme de Sévigné rejoint les critiques de son temps qui se sont prononcés contre les romans trop longs et elle aurait certainement souscrit à l'avis de du Plaisir qui ressent de «l'aversion» pour la «prodigieuse longueur» (COULET, $1968: 88$ ) des romans.

Outre les romans héroïques qu'elle aime par fidélité aux goûts de sa jeunesse, ce sont les romans parodiques, burlesques qu'elle cite volontiers quand la situation décrite dans la lettre s'y prête.

Ainsi la marquise mentionne deux fois Le berger extravagant de Charles Sorel publié en 1633 sous le titre de L'Anti-Roman', l'ouvrage où l'auteur se moque des romans, des aventures et des héros romanesques. Le personnage principal, Lysis, devient fou ayant lu trop de romans et se prend pour un berger de L'Astrée. Cet ouvrage est parodique et caricatural au point de remettre en cause sa propre existence.

C'est pourquoi Mme de Sévigné se réfère à Sorel quand elle parodie et ironise. Le 25 mars 1689, la marquise raconte à sa fille l'aventure amoureuse entre Mlle de Vaubrun et M. de Béthune, « le berger extravagant de Fontainebleau » (III, 91). Mme de Sévigné établit une analogie avec le roman de Sorel : 1'histoire réelle des amours de M. de Béthune correspond mal au modèle d'amant exploité par le roman héroïque, tout comme les aventures de Lysis sont une caricature de celles de Céladon. « Grand, maigre, un air de fou, sec, pâle. [...] Tel que je vous le figure, elle [Mlle de Vaubrun] l'aimait. [...] Cette petite fille de dix-sept ans a donc aimé ce don Quichotte, et hier il alla [...] enfoncer la grille du couvent avec une bûche et des coups redoublés. Il [...] la prend, l'emporte, la met dans un carrosse, la mène chez M. de Gêvres, fait un mariage sur la croix de l'épée, couche avec elle, et le matin [...] ils sont disparus tous deux et on ne les a pas encore trouvés » (III, 557558).

L'histoire s'est mal terminée: Béthune a été exilé et Mlle de Vaubrun placée dans un couvent où ses parents n'ont pu la voir que plusieurs années après. Mais ce qui plaît à Mme de Sévigné, c'est de raconter à sa fille une histoire qui se prête si bien au persiflage amusé.

\footnotetext{
${ }^{9}$ Le roman est cité pour la première fois en 1689 (III, 557). Pour la seconde fois, l'epistolière y fait allusion dans la lettre adressée à Pauline, en 1690 (III, 872).
} 
Celui qu'elle mentionne le plus souvent est Scarron qui, dans son Roman comique (1651), raille les romanciers dont les héros sont trop parfaits pour exister réellement. Cette perfection pousse le lecteur à désirer voir des personnages méchants. Scarron se moque aussi de certains procédés romanesques typiques pour les romans de Gomberville et de Georges et Madeleine de Scudéry (ADAM, 1973 : 559).

La marquise ne mentionne pas le roman lui-même, mais, soit elle fait allusion aux héros de Scarron, soit elle cite des formules qui ont marqué son esprit : «il [M. de Grignan] s'était tellement cassé le nez, et un peu la tête, et avait de si grand emplâtres que jamais La Rapinière ni Le Destin n'en portèrent de plus remarquables » (à Coulanges, 14. X. 1694, III, 1063). Chez Scarron, c'est le Destin qui « se deguisoit le visage d'un emplastre [...] et que se voyant travesty par accident, il avoit voulu oster aussi la connoissance de son visage à quelques ennemis » (ADAM, 1973 : 539). Mme de Sévigné aime aussi parler de celui qui « n'est pas bien assuré sur ses pieds ». Dans le roman de Scarron, pendant une représentation de Bradamante de Robert Garnier, des comédiens absents ont été remplacés par deux gentilhommes. L'un d'eux «jouoit le Page du Duc Aymond et n'avoit que deux Vers à reciter en toute la Piece, c'est alors que ce vieillard s'emporte terriblement contre sa fille Bradamante de ce qu'elle ne veut point épouser le fils de l'Empereur estant amoureuse de Roger : le Page dit à son Maistre :

Monsieur, rentrons dedans; je crains que vous tombiez,

Vous n'estes pas trop bien asseuré sur vos pieds.

Ce grand sot de Page, encore que son rôlle fust aisé à retenir, ne laissa pas de le corrompre et dit de fort mauvaise grace en tremblant comme un criminel :

Monsieur, rentrons dedans; je crains que vous tombiez,

Vous n'estes pas trop bien asseuré sur vos jambes.

Cette mauvaise rime surprit tout le monde. Le Comedien qui faisoit le personnage d'Aymond s'en esclatta de rire et ne put representer un vieillard en colere. Toute l'assistance n'en rit pas moins [...]. » (ADAM, 1973 : 681).

Mme de Sévigné termine par cette citation le passage dans lequel elle raconte comment son filleul s'est fait soigner « un petit mal des pieds » par un médecin qui lui « proposa, comme un remède anodin, de lui arracher de vive force les deux ongles des doigts où il avait mal, tout entiers, avec la racine, afin, disait-il, que cette incommodité ne revînt plus » (17. IV. 1671, I, 266). Une autre fois, elle le dit à propos du Chevalier de Grignan qui «croit toujours aller à Fontainebleau, mais il n'est pas encore trop bien assuré sur ses jambes. Il a pris une médecine dont il est content » (22. X. 1688, III, 375). Les contextes dans lesquels Mme de Sévigné cite le Roman comique montrent qu'elle appréciait non seulement les situations romanesques présentes chez Scarron (les duels, les enlèvements, les identités dissimulées, les hasards incroyables), mais qu'elle voyait aussi leur attrait grotesque.

Scarron prend pour héros de son roman des comédiens ambulants, Boileau, dans son Dialogue des héros de roman (commencé en 1664, achevé vers 1672 et publié en 1713, après la mort des auteurs de romans baroques), met en scène les principaux personnages des romans de La Calprenède et de Mlle de Scudéry et se moque sans pitié des auteurs qui ont fait «des Heros les plus considérables de 
l'Histoire des Bergers tres frivoles, et quelquefois meme des Bourgeois encore plus frivoles que ces Bergers » (BOILEAU, 1966: 444). Boileau trouve ces romans puérils, longs, parlant exclusivement de l'amour et cela dans un langage précieux et ridicule. Il avoue pourtant en avoir lu avec plaisir dans sa jeunesse. Il dit donc la même chose que Mme de Sévigné quand il estime que c'est le jeune âge qui pousse à ce genre des lectures. La différence, c'est que la marquise continue à succomber aux charmes des romans et même quand elle ne les lit plus, elle leur trouve quelques qualités, tandis que Boileau en fait la cible de ses critiques.

La marquise mentionne les œuvres de Boileau-Despréaux, parle longuement de sa fonction d'historiographe du roi et avant tout le traite comme une autorité en matière de jugements littéraires. Quand, le 16 mars 1672, elle critique Bajazet de Racine et loue «notre vieil ami Corneille », elle constate que " Despréaux en dit encore plus » (16. III. 1672, I, 459). Ménage et Chapelain ne s'aimaient pas du tout, Boileau ridiculisait Chapelain dont il trouvait les vers mauvais, mais la marquise les considérait néanmoins tous trois comme des maîtres en théorie littéraire, tout en sachant très exactement quels étaient leurs rapports : « Despréaux vous ravira par ses vers. Il est attendri pour le pauvre M. Chapelain; je lui dis qu'il est tendre en prose, et cruel en vers » (15. XII. 1673, I, 641).

L'Art poétique a paru en 1674. Mme de Sévigné y fait allusion bien avant, le 9 mars 1672 , ce qui prouve qu'elle avait connaissance des œuvres de son temps avant la publication et qu'elle les recevait souvent par avance, comme les romans de Mlle de Scudéry. En décembre 1673, elle affirme avoir écouté la lecture du traité de Boileau : " Je dînai hier avec Monsieur le Duc, M. de La Rochefoucauld, Mme de Thianges, Mme de La Fayette, Mme de Coulanges, l'abbé Têtu, M. de Marsillac et Guilleragues, chez Gourville. [...] On écouta la Poétique de Despréaux, qui est un chef-d'œuvre » (15. XII. 1673, I, 640).

Quand l'épistolière mentionne la querelle des anciens et des modernes, elle ne peut pas omettre le nom de Boileau-Despréaux. Le 15 janvier 1690, elle transmet à Mme de Grignan le contenu d'une longue discussion entre Boileau, Corbinelli et le P. Bourdaloue : «On parla des ouvrages des anciens et des modernes. Despréaux soutint les anciens, à la réserve d'un seul moderne qui surpassait, à son goût, et les vieux et les nouveaux » (15. I. 1690, III, 811). Dans le long passage que l'épistolière consacre à cet échange entre les érudits, Boileau finit par nommer ce seul moderne qui trouve grâce à ses yeux : Pascal - ce qui entraîne une très vive polémique au cours de laquelle se manifeste le caractère violent et impulsif de l'auteur de l'Art poétique. Il est certain que la marquise ne suivait pas le déroulement de la querelle par pur intérêt littéraire (celui d'un écrivain ou d'un critique), mais parce qu'elle était proche des jansénistes. Elle lisait Pascal avec beaucoup d'attention et elle adhérait à bon nombre de ses idées. Elle voulait donc savoir ce que les autres pensaient de sa doctrine. Le désir d'être au courant incitait la marquise à observer les comportements des critiques face à ce conflit de goûts, de sensibilité, de traditions et aussi théologique.

D'autres érudits encore, importants pour la théorie romanesque au XVII siècle, sont présents dans les lettres, tels le P. Le Bossu et son Traité sur le poème épique (II, 414), Desmarets de Saint-Sorlin ou Pierre-Daniel Huet qui figure dans la Correspondance à l'occasion de sa critique de la philosophie de Descartes, 
critique à laquelle « bien des gens répondront» (15. VI. 1689, III, 618), prévoit à juste titre la marquise.

Mme de Sévigné parle aussi de Segrais qu'elle appelle «son ami » (5. V. 1689, III, 592) et évoque les soirées passées en sa compagnie (8. I. 1672, I, 412 ; 4. III. 1672, I, 450). C'est à lui que Huet dédie sa Lettre de l'origine des romans, c'est sous son nom que paraît Zaïde ${ }^{10}$ et c'est lui aussi qui collabore à la rédaction de la Princesse de Clèves. Ses Nouvelles françoises (1657) sont un dialogue entre la princesse Aurélie et cinq autres dames. Segrais fait discuter seulement les femmes, car le goût des romans leur est «quasi naturel». Il réfléchit sur les aspects contradictoires du roman, à savoir, comment concilier l'histoire, le réel avec le goût des aventures extraordinaires.

La marquise parle de lui pour la première fois dans sa lettre du 20 mars 1671 : « Je crois que vous savez que Mademoiselle a chassé Guilloire. Le pauvre Segrais ne tient à guère. C'est qu'ils ont témoigné trop librement leurs sentiments sur $\mathrm{M}$. de Lauzun » (20. III. 1671, I, 194). Segrais était au service de Mademoiselle de Montpensier depuis vingt-quatre ans. Comme il lui déconseillait de revoir Lauzun après son mariage manqué, il fut chassé de chez elle et il habita chez Mme de La Fayette de 1671 à 1676. Cela explique en partie leur collaboration littéraire.

Le $1^{\text {er }}$ avril 1671, Mme de Sévigné a en vain essayé de convaincre Mademoiselle de ne pas chasser Segrais : « Je lui ai parlé de Segrais, à la romaine, prenant son parti, mais elle n'est pas traitable sur ce qui touche à neuf cents lieues près de la vue d'un certain cap, d'où l'on découvre les terres Micomicon » (1. VI. 1671, I, 205).

C'est surtout l'esprit et le sens de l'humour que Mme de Sévigné aime chez Segrais. Le 4 mars 1672, elle raconte à sa fille comment il s'est moqué de la marquise de Gouville : «A propos des étoiles, la Gouville était l'autre jour chez la Saint-Loup [...] et parlait de son étoile ; enfin que c'était son étoile qui avait fait ceci, qui avait fait cela. Segrais se réveilla comme d'un sommeil, et lui dit: « Mais, madame, pensez-vous avoir une étoile à vous toute seule? Je n'entends que des gens qui parlent de leur étoile! [...] Savez-vous bien qu'il n'y en a que mille vingt-deux? Voyez s'il peut y en avoir pour tout le monde ». Il dit cela si plaisamment et si sérieusement, que l'affliction en fut déconcertée » (4. III. 1672, I, 449-450).

«L'esprit vif et enjoué » (SOMAIZE, 1856 : 221), Mme de Sévigné ne peut qu'apprécier la plaisanterie de Segrais. Les lettres contiennent beaucoup d'anecdotes qui prouvent que l'épistolière est tout particulièrement sensible à l'intelligence et à l'humour de ceux qu'elle fréquente. Formée par ses amisérudits, par les conversations et les lectures, elle « a une promptitude d'esprit la plus grande du monde à connaître les choses et à en juger » (SOMAIZE, 1856 : 221).

Mais, comme l'on vient de voir, la marquise ne se comporte jamais en critique ou expert de la littérature ou de la théorie critique. C'est aux hommes faisant profession de critiques (Chapelain, Ménage, Rapin, Bouhours) ou considérés comme une autorité dans le domaine (Corbinelli, Bussy) qu'elle laisse cette tâche. Les connaissances littéraires de l'épistolière affleurent seulement à travers de

\footnotetext{
${ }^{10}$ Dans l'édition de 1700, la Lettre à M. de Segrais de l'origine des romans et Zaïde ont été publiés ensemble, cité d'après COULET (1968: 181)
} 
courtes remarques isolées. Elles constituent parfois une invitation à la discussion, mais le plus souvent les allusions aux romans servent à illustrer, parodier ou agrémenter l'histoire relatée.

L'on peut aussi se demander si les préceptes théoriques du genre romanesque n'ont pas influencé le style de Mme de Sévigné dont les courtes narrations obéissent aux lois imposées par les romanciers. Parfaitement assimilées, les idées sur les romans font partie intégrante de la culture humaniste de Mme de Sévigné.

La connaissance des théoriciens et de leurs théories, la fréquentation assidue des cercles où l'on en débatait n'empêchaient pas la marquise de fonder ses jugements littéraires aussi et surtout sur le plaisir que lui procure la lecture. Son goût des romans, même quand elle en a honte et qu'elle trouve leur style médiocre, a sa source dans les premiers romans héroïques qu'elle a lus, dans ceux de Mlle de Scudéry et dont elle a fait sienne l'esthétique. Mme de Sévigné savoure le climat de ces romans et évite systématiquement une analyse fondée sur les principes au profit de celle qui saisit les éléments subtils dont dépendent les agréments d'un ouvrage.

\section{BIBLIOGRAPHIE}

\section{Bibliographie primaire :}

Mme de SÉVIGNÉ, (1972-1978), Correspondance, texte établi, présenté et annoté par Roger Duchêne, Paris, Bibliothèque de la Pléiade, Gallimard, 3 vol.

ADAM Antoine, (1ère éd. 1958, 1973), Les romanciers du XVII ème siècle, Paris, Gallimard, Bibliothèque de la Pleiade.

Berger Günter, (éd.), (1996), Pour et contre le roman. Anthologie du discours théorique sur la fiction narrative en prose du XVIIe siècle, Papers on Seventeenth Century Literature, Biblio 17, $\mathrm{n}^{0} 92$.

BoILEAU Nicolas, (1966), Euvres complètes, Paris, Gallimard, Bibliothèque de la Pléiade. Chapelain Jean, (1999), De la lecture des vieux romans, présentation, notes et établissement du texte Jean-Pierre Cavaillé, Paris, Zanzibar.

AubIGNAC François Hédelin abbé d' (1664), Macarise ou la Reyne des Isles fortunées, préface, Paris, pp. 3-213.

GeGoU Fabienne (éd.critique), (1971), Lettre-traité de Pierre-Daniel Huet sur l'origine des romans : édition du tricentenaire, 1669-1969. Suivie de la Lecture des vieux romans, par Jean Chapelain, Paris, Nizet.

Mme de LA FAYETTE, (1942), Correspondance, éditée d'après les travaux d'André Beaunier, Paris, Gallimard.

SCUDÉRY Madeleine de, (1654), Artamène ou Le Grand Cyrus, Paris, page internet.

SCUDÉRY Madeleine de, (1682), Conversations morales, «De la manière d'écrire une fable », Amsterdam.

SEGRAIS Jean Regnault de, (1992), Les nouvelles françaises ou les divertissements de la Princesse Aurélie, texte édité, présenté et annoté par ROGER Guichemerre, Paris, Société des Textes Français Modernes.

SomaIzE Antoine de, (1856), Le Grand Dictionnaire des Précieuses, Paris, 2 vol.

Bibliographie secondaire :

COUlet Henri, (1968), Le roman jusqu'à la Révolution, Paris, A. Colin, Coll. «U », 1er vol.

DUCHENE Roger, (1982), Madame de Sévigné ou la chance d'être femme, Paris, Fayard. 
DuCHENE Roger, (1996), Naissance d'un écrivain: Madame de Sévigné, Paris, Fayard.

Kulesza Monika, (1998), "Le théâtre dans les lettres de Madame de Sévigné », in Acta Philologica (25), «Literatura i jej pogranicza », Wydział Neofilologii UW, p. 61-82.

MAGNE Bernard, (1973), « Humanisme et culture féminine au XVII s. d'après les lettres de Madame de Sévigné », Marseille n ${ }^{0}$ 95, p. 37-41.

VIALA Alain, « Un jeu d'images : amateur, mondaine, écrivain? », Europe, janvier-février 1996, p. 57-69. 


\section{ABSTRACT}

Mme. de Sevigné was neither a novel writer nor a literary critic. She was an erudite and a woman of the world, frequenting salons where disputes and discussions, often about literature, were conducted.

In the $17^{\text {th }}$ century, the novel was by no means treated as a literary genre since Aristotle never mentioned it in his Poetics. But it was the novel that was the most frequently read. The times of Descartes had to produce rules of the genre that never had them before.

In my article, I have presented comments regarding novel writing, known to Mme. de Sevigné, usually described in Prefaces to works known to the Marchioness. I have analysed fragments of her letters where Mme. de Sevigné wrote about erudites, engaged in writing about the theory of the novel, about novel writers and their concepts on principles of novel writing.

The first group includes the Marchioness' friends Gilles Ménage and Jean Chapelain, both authorities in the field of literature. Their opinions on the works discussed in her letters counted the most for Mme. de Sevigné. Furthermore, I have presented novels, read by Mme. de Sevigné, whose authors, in Prefaces to their works, shared their opinions on the principles of the novel writing with their readers, e.g. Georges and Madeleine de Scudéry, as well as Charles Sorel and Paul Scarron. The latter are mentioned by Mme. de Sevigné whenever she talked about grotesque characters of novels or their behaviour. Mme. de Sevigné mentioned many others such as Pierre-Daniel Huet or Nicolas Boileau. L'Art poétique was, even before publication, a well-known work, widely discussed in the salons and admired.

Although Mme. de Sevigné knew theoretical works, she nevertheless, judged the novels by the pleasures their reading gave her. 